\title{
Möbius and problems of inversion
}

Who says that the theory of numbers is strictly academic? An old theorem due to Möbius has unexpectedly proved to be a way of solving physical problems of inversion that may have important applications.

THE belief that pure mathematics is only fortuitously useful is widely shared, even by mathematicians. So why is the practice of science increasingly mathematical? There are two explanations. First, good luck; mathematicians do so many things that some of them must be useful. Second, psychology; the purest mathematicians have an unconscious sense of the urgent problems of the real world, and shape their interests accordingly.

There is also, of course, the Hilbert phenomenon. David Hilbert (1863-1943), whose first claim on public attention was his demonstration that Euclid's axioms are not the self-consistent structure they had seemed for two millennia, afterwards bent his talent to the solution of physicists' problems, founding a tradition now carried on by people such as Atiyah (Oxford) and L. D. Fadeev (Moscow).

But there may also be something in the view that all mathematics is potentially useful, that mathematicians have littered the literature with gems of technique whose usefulness is waiting to be discovered. For did not the nineteenth century's preoccupation with continued fractions prove useful, if briefly, in field theory? Did not Hamilton's quaternions, devised as a means of completing the algebra of vector quantities by the definition of a quotient, turn out to have interesting connections with the spinor algebra of relativistic quantum mechanics?

That is the spirit in which one should celebrate the use now discovered for a recondite contribution to the theory of numbers by Möbius (1790-1846), best known for the topological conundrum called the Möbius strip. Among other things, Möbius noted a simple inverse relationship between functions in number theory, which takes the following form.

First, take some function $f(n)$ of the integer variable $n$ and another function $F(n)$ defined as $\Sigma f(d)$, where the summation runs only over the divisors of $n, 1$ and $n$ included. Then, according to Möbius, it is possible to invert the functional relationship into the simple $f(n)=$ $\Sigma \mu(d) F(n / d)$ where the sum again runs over all divisors of $n$, and where the coefficients $\mu(d)$, all either zero or \pm 1 , reflect the prime composition of $d$.

Briefly, $\mu(1)=1$, and $\mu(d)=0$ except when $d$ is either a prime number or a composite number which is the product of, say, $r$ distinct primes, when it has the value $(-1)^{r}$. One can while away hours on aircraft journeys verifying that the inversion works. To show that $\Sigma \mu(d)$ differs from zero (with the same restrictions on the summation) only when $n=1$ requires more ingenuity; one has to express $n$ as a formal product of prime numbers and then show that the sum is $(1-1)^{n}$.

What use is this? Inversion is the key word. Physical problems are most often inversion problems - inferring the velocity profile in the Earth's crust from seismic signals, for example, or the linear distribution of interplanetary electron density from the measured Farady rotation of the plane of polarization of a radio signal from a satellite of some kind. And now Nan-xian Chen, from the Technical University at Beijing, has turned Möbius's inversion theorem to practical use by the exercise of more than a little ingenuity (Phys. Rev. Lett. 64, 1193; 1990). The work was done when Chen was at the International Institute of Theoretical Physics at Trieste.

The trick is to show that the integers in Möbius's inversion formula can be replaced by continuous variables, which hangs on a proof that the infinite series arising do indeed converge. The essence of Chen's paper is the proof that if $A(\omega)$ $=\Sigma B(\omega / n)$, where the summation extends from $n=1$ to infinity, the inverse is given by $B(\omega)=\Sigma \mu(n) A(\omega / n)$, with the same summation rule.

Chen proves his point by demonstration, with a string of examples only otherwise solved with difficulty. One of them, a new result, is neat enough at least to illustrate the potential power of the method. Suppose that the vertices of an infinite one-dimensional lattice are all occupied with interacting atoms, so that any one of them experiences a potential $V(x)=$ $\Sigma v(n x)$, where the summation in $n$ is from 1 to infinity and $v(x)$ is the elementary pair-wise interaction. How, one might ask, can that be related explicitly to $V(x)$, which might be measurable? What the inversion gives is simply $\Sigma \mu(n) V(n x)$ or, more explictly, $V(x)-V(2 x)-V(3 x)$ $-V(5 x)+V(6 x) \ldots$.

A more interesting example is that of how it may be possible accurately to infer the frequency distribution of the vibrational states of a solid lattice, say $g(v)$, from measurements of specific heat at constant volume. Apart from numerical factors involving Planck's constant and Boltzmann's constant, the latter is simply the integral from zero to infinity of $v^{2} g(v)$ weighted by an appropriate Planck-Boltzmann factor allowing for the increased excitation of higher frequencies with increasing temperature. At low temperatures, it is usually feasible to represent the measured specific heat as a power series in the temperature $T$, beginning with a cubic term.

As if pulling a rabbit out of a hat, Chen relates $g(v)$ directly to the measured coefficients in the power series representing the specific heat. As a reminder that the theory of numbers lies at the basis of all this sleight of hand, values of Riemann's zeta function for integral multiples of 2 appear throughout, which means that they can be written as Bernoulli numbers. Chen's other example is that of inferring the temperature distribution of a composite black body from measurements of its power output, which he says is a problem of current interest in remote sensing.

Where will all this lead? The ideal, for Chen, would be that somebody should put a previously unsolved problem through the new Möbius mill. It will be interesting to see which problems first suggest themselves as candidates. A more demanding question is whether it will be feasible to extend the trick to problems that are not simply one-dimensional. On the face of things, that might seem a mere formality, but it takes only a little scribbling to run into problems essentially tied up with the multiple connectedness of all but onedimensional spaces. But that should not be a discouragement; rather, a challenge. It is fair to guess that, with Chen's proof that even Möbius has something to tell the modern world, a small army will now be scouring the literature of the theory of numbers in the hope of finding other useful tools in what may have been unjustly regarded as a backwater.

There is no shortage of material. Chen himself quotes the fifth edition of the classic Theory of Numbers by G. H. Hardy and E. M. Wright (Clarendon, Oxford; 1979), which does indeed tell all about the Möbius inversion without hinting that it may have physical application. Alongside that is a neat proof that Ramanujan's sum, defined as $\Sigma \mathrm{e}^{(2 \pi i h m / n)}$ with the sum in $h$ running only over values less than $n$ and prime to it, is also $\Sigma \mu(n / d) d$, where $d$ is both a divisor of $m$ and $n$. Surely, one is bound to ask, there must be some value in that?

John Maddox 